\title{
Estrategias actuales en el manejo de las secreciones traqueobronquiales
}

\section{Current strategies in the management of airway secretions}

\author{
Arturo Cortes-Telles, ${ }^{*}$ José Luis Che-Morales, ${ }^{\ddagger}$ Diana Lizbeth Ortiz-Farías*
}

*Hospital Regional de Alta Especialidad de la Península de Yucatán;

‡Unidad Médica de Atención Ambulatoria. IMSS, Mérida, Yucatán, México.

\begin{abstract}
RESUMEN. El aumento de la producción de moco es común en pacientes con enfermedades respiratorias, asimismo, en la población en general como resultado de la exposición a partículas irritantes e infecciones. La hipersecreción del moco en las vías respiratorias contribuye al deterioro del aclaramiento mucociliar, promueve la mucoestasis y, potencialmente, obstrucción de la vía aérea; esta acumulación continua de moco compromete el trabajo respiratorio con importante gasto energético afectando de manera negativa en la calidad de vida de las personas. Sin embargo, la investigación sobre los beneficios de las intervenciones utilizadas para controlar la hiperproducción de moco es escasa. El propósito de esta revisión es describir las diferentes medidas (farmacológicas y no farmacológicas) que existen en la actualidad para el manejo de las secreciones respiratorias.
\end{abstract}

Palabras clave: Fármacos mucoactivos, aclaramiento mucociliar, dispositivos mecánicos, aerosol terapia, mecanismos de la tos.

\section{INTRODUCCIÓN}

En individuos sanos, diferentes mecanismos intervienen para mantener un ambiente de esterilidad en la vía respiratoria e incluyen: 1) barreras mecánicas (moco), 2) integridad del aclaramiento mucociliar y 3) la tos. La interacción de cada uno de los componentes forma parte de un proceso fisiológico con el propósito de prevenir el desarrollo de infecciones. ${ }^{1}$

Diariamente se producen en promedio $50 \mathrm{ml}$ de moco que se encarga de recubrir la vía respiratoria y es regulado mediante un ritmo circadiano; asimismo, se intercambia a frecuencia constante mediante el movimiento ciliar. Dado que el moco posee propiedades antioxidantes, antimicrobianas

Correspondencia:

Dr. Arturo Cortes-Telles

Hospital Regional de Alta Especialidad de la Península de Yucatán.

Yucatán, Mérida, México.

Correo electrónico: dr_morenheim@hotmail.com

Trabajo recibido: 28-II-2019; aceptado: 10-VI-2019.
ABSTRACT. Increased mucus production is common in patients with respiratory diseases, also in the general population as a result of exposure to irritating particles and infections. An increase in the amount of mucus in the respiratory airway contributes to the deterioration of mucociliary clearance, promotes muco-stasis and, potentially, obstruction of the airway. The continuous accumulation of mucus compromise work of breathing with significant energy expenditure, negatively affecting the quality of life. Research on the benefits of interventions used to control mucus overproduction is scarce. The purpose of this review is to describe the different measures (pharmacological and non-pharmacological) that currently exist for the management of airway secretions.

Keywords: Mucoactive agents, mucociliary clearance, mechanical devices, aerosol therapy, cough mechanism.

y antiproteasas, tiene la capacidad de atrapar y neutralizar partículas, bacterias y virus; en conjunto, se genera una mezcla de mayor volumen que sobrepasa la capacidad normal de remoción del movimiento ciliar e interviene el reflejo de la tos para expulsarlo. ${ }^{2}$ Lo anterior, es el ejemplo clásico que denota la interacción y trascendencia de los mecanismos naturales de defensa del aparato respiratorio (figura 1).

\section{Desequilibrio de la vía respiratoria}

Por lo regular, dos condiciones fisiopatológicas desequilibran los mecanismos de defensa innatos en la vía respiratoria: 1) procesos inflamatorios y 2) procesos infecciosos. ${ }^{3}$ En ambos contextos, se desarrolla una respuesta antiinflamatoria intensa acumulando productos de destrucción bacteriana, entre ellos: filamentos de actina y ADN de neutrófilos; asimismo, remanentes derivados de apoptosis celular y microorganismos; en conjunto, favorecen el aspecto purulento y perpetúan los cambios nocivos en la reología del moco (aumento en la viscosidad y espesor) contribuyendo con mayor dificultad para su expectoración. ${ }^{4}$ 
Neumol Cir Torax. 2019; 78 (3): 313-323

Diversas enfermedades respiratorias agudas y crónicas favorecen el acumulo de secreciones a través de cualquiera de los siguientes mecanismos: incremento en la producción de moco, alteración en el transporte mucociliar o a una tos ineficiente. ${ }^{4}$ Del mismo modo, existen escenarios clínicos que pueden alterar de forma grave los mecanismos naturales de defensa, incrementando el riesgo de colonización bacteriana y potencialmente el desarrollo de procesos infecciosos (v.gr., neumonía), entre ellos, intubación orotraqueal y apoyo con ventilación mecánica. ${ }^{5}$

En conclusión, el manejo inadecuado de las secreciones en el aparato respiratorio contribuye con el desarrollo de múltiples morbilidades, incluyendo: taponamientos mucosos, atelectasias obstructivas y el desarrollo de lesión pulmonar. Por lo tanto, es aconsejable emplear medidas para mejorar el aclaramiento de la vía respiratoria.

\section{MANEJO FARMACOLÓGICO}

\section{Agentes mucolíticos, mucocinéticos y mucorreguladores}

El estímulo lesivo en la vía respiratoria desencadena un incremento en el volumen de secreción por las glándulas mucosas que puede sobrepasar la tasa de extracción del aparato mucociliar. ${ }^{6} \mathrm{Si}$ en paralelo existen cambios en las características biofísicas del moco favoreciendo una inadecuada proporción entre viscosidad y elasticidad con mayor adhesividad hacia el epitelio respiratorio se compromete su remoción. ${ }^{6,7}$

Cabe destacar que durante muchos años se han generado fármacos que alteran las propiedades viscoelásticas del moco promoviendo su aclaramiento. A éstos, se les denomina fármacos mucoactivos e incluyen: 1) expectorantes, 2) mucolíticos, 3) mucorreguladores y 4) mucocinéticos (tabla 1).?

\section{Expectorantes}

Se define como expectorante a todo agente farmacológico que mejora la capacidad para expulsar secreciones procedentes del árbol traqueobronquial. ${ }^{7}$ El mecanismo de acción preciso es incierto, se consideran secretagogos y favorecen el incremento del volumen de agua en el epitelio de la vía respiratoria; asimismo, durante un período de tiempo se consideró que actuaban como irritantes de los receptores vagales favoreciendo una menor viscosidad del moco, mecanismo actualmente sin validez. ${ }^{8}$

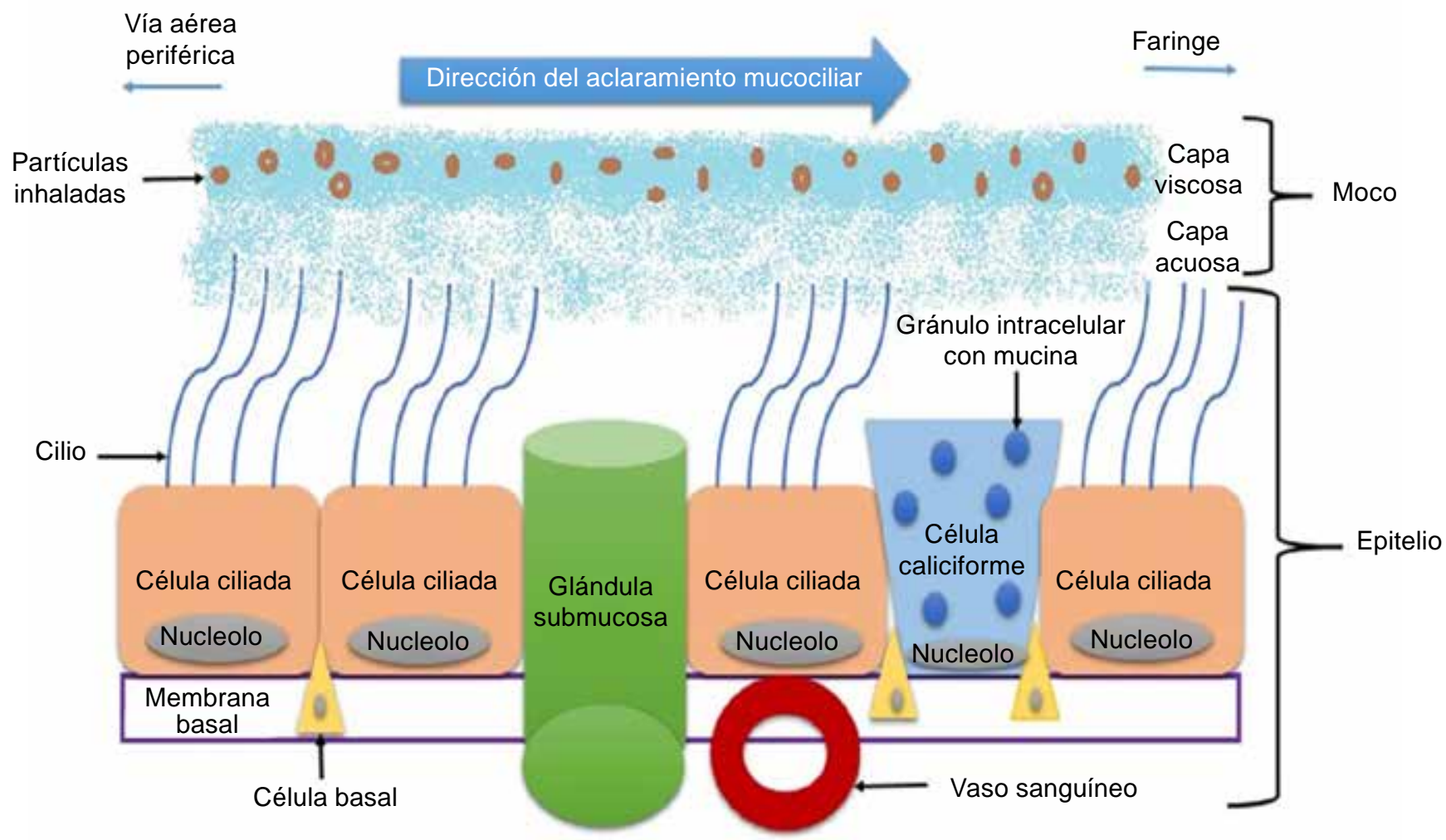

Figura 1: Epitelio respiratorio. El moco es producido por las células caliciformes y las glándulas submucosas. El aclaramiento mucociliar, es el proceso por el cual se elimina el moco de las vías aéreas inferiores hacia la faringe por un proceso de barrido que realizan los cilios. 
Tabla 1: Clasificación de fármacos mucoactivos y mecanismo de acción identificado.

\begin{tabular}{|c|c|}
\hline Fármaco & Mecanismo de acción potencial \\
\hline $\begin{array}{l}\text { Mucorreguladores } \\
\text { Anticolinérgicos } \\
\text { Glucocorticoides } \\
\text { Macrólidos }\end{array}$ & $\begin{array}{l}\text { Disminuyen volumen de secreción } \\
\text { Disminuyen inflamación de vía aérea y secreción de mucina } \\
\text { Disminuyen inflamación de vía aérea y secreción de mucina }\end{array}$ \\
\hline $\begin{array}{l}\text { Mucolíticos clásicos } \\
\text { N-acetilcisteína }\end{array}$ & $\begin{array}{l}\text { Disuelve los puentes disulfuro que unen los polímeros de mucina. Antioxidante y antiinflamatorio. El } 70 \% \text { de la } \\
\text { carga farmacológica se elimina en menos de } 15 \text { minutos }\end{array}$ \\
\hline $\begin{array}{l}\text { Péptidos mucolíticos } \\
\text { Alfa-Dornasa } \\
\text { Timosina } \beta_{4} \\
\end{array}$ & $\begin{array}{l}\text { Hidroliza polímeros de ADN y disminuye la longitud del ADN. Disminuye viscosidad del moco en vía aérea } \\
\text { Despolimeriza los filamentos de actina }\end{array}$ \\
\hline $\begin{array}{l}\text { Otros mucolíticos } \\
\text { Inhibidores de Tirocin Cinasa } \\
\text { Estatinas } \\
\text { P3001 }\end{array}$ & $\begin{array}{l}\text { Inhiben EGFR (receptor del factor de crecimiento epidérmico) disminuyendo la producción de mucina y la hiper- } \\
\text { plasia de células caliciformes; aún en fase experimental } \\
\text { Disminuyen la hiperplasia de células caliciformes e inhibe la expresión del RNAm de MUC5AC } \\
\text { Rompen los puentes disulfuro de las mucinas MUC5AC y MUC5B y se elimina seis veces más lento que la N- } \\
\text { acetil-cisteína. Está en fase experimental }\end{array}$ \\
\hline $\begin{array}{l}\text { Mucocinéticos } \\
\text { Broncodilatadores } \\
\text { Surfactante }\end{array}$ & $\begin{array}{l}\text { Mejoran el aclaramiento de tos al incrementar el flujo espiratorio } \\
\text { Disminuye la viscosidad del moco/esputo }\end{array}$ \\
\hline
\end{tabular}

Bajo el concepto de expectorantes, por lo general son empleados la hidratación oral, fármacos que contienen yodo, guaifenesina y recientemente, modificadores de canales iónicos (v.gr., agonistas purinérgicos). Más aún, otros fármacos como la solución salina hipertónica y el manitol, son considerados expectorantes, dado que favorecen mayor volumen de agua en la vía respiratoria.

Sin embargo, los expectorantes no tienen ningún efecto sobre la frecuencia del latido ciliar; incluso, la guaifenesina ha mostrado tener efectos tóxicos sobre el epitelio respiratorio al disminuir el latido ciliar. ${ }^{9}$ En adición, los derivados de yodo y la hidratación simple no han demostrado ser clínicamente efectivos como expectorantes en ensayos controlados. $^{9-11}$

\section{Mucolíticos}

El mecanismo farmacológico de los mucolíticos es modificar las propiedades biofísicas de las secreciones y disminuir la viscosidad. Bajo este concepto las secreciones pueden movilizarse en conjunto con el mecanismo de la tos. ${ }^{7}$ Por lo tanto, no deben administrarse de forma simultánea con inhibidores del reflejo tusígeno (v.gr., dextrometorfano, ben- zonatato, dropropizina, etc.). Los mucolíticos se clasifican como: 1) clásicos, aquellos que degradan los polímeros de mucina y 2) péptidos mucolíticos, cuando tienen la capacidad de degradar los polímeros de ADN y filamentos de actina. $^{3}$

El mucolítico clásico empleado por lo general en la práctica clínica es $\mathrm{N}$-acetilcisteína. Dado que adicionalmente tiene propiedades antiinflamatorias y antioxidantes se utiliza en patologías obstructivas crónicas y enfermedades fibrocicatriciales del pulmón. ${ }^{12,13}$ Se ha documentado que al administrarse en forma de aerosol disminuye la viscosidad de las secreciones; no obstante, en pacientes con fenotipo de bronquitis crónica, no genera un cambio en la función pulmonar o el volumen de esputo, más aún, puede ocasionar broncoconstricción e inhibir la frecuencia del latido

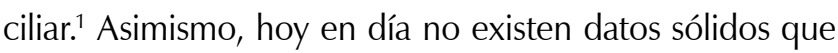
demuestren una mejoría en el manejo de secreciones, calidad de vida y frecuencia de exacerbaciones en enfermedades obstructivas. ${ }^{14,15}$

Recién, Ehre y colaboradores demostraron la eficacia in vivo de la molécula P3001 en ratones mutados con sobreexpresión del gen de canales de sodio (modelo similar a fibrosis quística). El fármaco tuvo efecto sobre las 
Neumol Cir Torax. 2019; 78 (3): 313-323

mucinas MUC5AC y MUC5B que usualmente se elevan en mayor o menor proporción en enfermedades como asma, enfermedad pulmonar obstructiva crónica (EPOC) y fibrosis quística; el mecanismo propuesto es a través de la ruptura de los enlaces disulfuro del moco alterando sus propiedades bio-físico-químicas y sin toxicidad aparente tanto in vivo como in vitro. Los efectos son similares a los demostrados por la N-acetilcisteína, aunque, esta última se absorbe de forma rápida (70\% del fármaco en menos de 15 minutos) en el epitelio respiratorio por lo cual carece de acción sostenida y explica de modo parcial la falta de éxito en ensayos clínicos previos. ${ }^{16}$

Por otro lado, entre los péptidos mucolíticos, alfa-dornasa, es el único aprobado para el manejo de secreciones en pacientes con fibrosis quística (FQ). La administración de alfa-dornasa vía nebulizada mejora la viscosidad y adhesividad del moco; asimismo, el volumen espiratorio forzado durante el primer segundo en pacientes con FQ. ${ }^{17}$

Cabe destacar que existen varios fármacos con acción mucolítica que aún se encuentran en fase de estudio, entre ellos figuran los inhibidores de tirosina cinasa, estatinas e inmunoterapia, estos medicamentos han demostrado en ratones disminuir la hiperplasia de las células caliciformes, por ende, disminuyen la producción de moco. No obstante, hasta el momento no existen estudios que demuestren que tenga la misma eficacia en pacientes con enfermedades respiratorias..$^{18,19}$

\section{Mucocinéticos}

Comúnmente referidos como fármacos que mejoran el aclaramiento de la tos al actuar de forma directa en el latido ciliar. Es importante mencionar que no se ha demostrado un efecto consistente sobre el aclaramiento mucociliar en pacientes con historia de enfermedad pulmonar crónica. ${ }^{7}$ Entre los medicamentos con capacidad mucocinética se encuentran: a) broncodilatadores ( $\beta$-2 agonistas); b) nucleótidos tricíclicos, c) ambroxol y d) macrólidos. ${ }^{3}$

Los $\beta$-2 agonistas poseen el mejor efecto mucocinético. Dicho efecto se alcanza al disminuir la resistencia de la vía aérea favoreciendo un incremento en el flujo espiratorio que moviliza con mayor facilidad las secreciones. ${ }^{20}$ Estos fármacos están indicados en los pacientes que tengan evidencia de obstrucción al flujo aéreo (v.gr., EPOC, asma, bronquiectasias), riesgo de atelectasia (v.gr., síndrome de reposo prolongado).

Los macrólidos han sido impulsados como medicamentos adyuvantes al tratamiento de diversas enfermedades respiratorias caracterizadas por inflamación y obstrucción en la vía aérea. ${ }^{21} \mathrm{~A}$ pesar de no precisar el mecanismo de acción, tienen efectos específicos que potencialmente promueven la mejoría del aclaramiento mucociliar. Entre ellos se han descrito: 1) inhibición en la quimiotaxis de neutrófilos; 2) regulación del tono neural en la vía aérea y 3) modular la contracción del músculo liso bronquial. ${ }^{22}$ El beneficio a largo plazo ha sido informado en un análisis reciente por Albert y colaboradores quienes concluyen que la adición de $250 \mathrm{mg} /$ día de azitromicina al tratamiento convencional durante 52 semanas disminuye el riesgo y la frecuencia de exacerbaciones en pacientes con EPOC. ${ }^{23}$

El empleo de otros fármacos con acción mucocinética como ambroxol o bromhexina administrados vía oral o nebulizada no han demostrado un beneficio contundente. ${ }^{3}$ A pesar de un informe reciente sobre la posible utilidad del ambroxol como antiinflamatorio y antioxidante, los autores consideramos que debe mejorarse la evidencia sobre la utilidad del fármaco en el manejo de secreciones mediante el desarrollo de ensayos controlados..$^{24,25}$

\section{Corticoesteroides inhalados}

El uso de corticoesteroides por vía inhalada maximiza los efectos en el tejido pulmonar. Clínicamente, se traduce con la mejoría de los síntomas respiratorios, menor requerimiento de esteroides sistémicos y mejoría de la función pulmonar. ${ }^{26}$

Si bien, el mecanismo de acción de los corticoesteroides inhalados (CEI) a nivel molecular no está definido con precisión, se conoce ampliamente la interacción con el factor nuclear- $\kappa \beta$ (NF- $\kappa \beta)$ que bloquea la transcripción genética. ${ }^{27}$ Por otro lado, en la vía respiratoria, los CEI disminuyen el número de células implicadas en la fisiopatología del asma; asimismo, inhiben la liberación de mediadores inflamatorios derivados de linfocitos, macrófagos y células del epitelio alveolar. Por último, los CEI parecen inhibir la función de las glándulas submucosas atribuyendo un efecto mucorregulador, no obstante, este mecanismo no ha sido del todo sustentado. ${ }^{20}$

\section{Solución salina $0.9 \%$ (SS0.9\%)}

La administración de SS0.9\% en la vía respiratoria forma parte del manejo rutinario de las secreciones traqueobronquiales en las unidades de cuidados intensivos (UCI). Al parecer dicha estrategia antecede al desarrollo de dispositivos generadores de humedad. En un inicio, el propósito de utilizar SS0.9\% era lubricar el paso del catéter de succión a través de la cánula orotraqueal. No obstante, en la práctica clínica convencional se señala con frecuencia que la justificación para administrar SS0.9\% en la vía respiratoria radica en: 1) movilizar las secreciones traqueobronquiales, 2) aumentar el estímulo tusígeno y 3) «fluidificar» las secreciones. A pesar de esto, se carece de evidencia sólida que respalde estas aseveraciones. ${ }^{28}$

Por otro lado, contamos con evidencia suficiente que describe los efectos deletéreos relacionados con el uso 
Neumol Cir Torax. 2019; 78 (3): 313-323

Tabla 2: Tipos de dispositivos mecánicos para manejo de secreciones.

\begin{tabular}{|l|l|}
\hline \multicolumn{1}{|c|}{ Dispositivo } & \multicolumn{1}{|c|}{ Mecanismo de acción y grado de recomendación USPSTF (U.S. Preventive Services Task Force) } \\
\hline $\begin{array}{l}\text { Positive Espiratory Pressure } \\
\text { (PEP) }\end{array}$ & $\begin{array}{l}\text { Presión positiva espiratoria. Mejora el drenaje de las secreciones, ya sea por incremento de la presión del gas } \\
\text { detrás del moco a través de ventilación colateral o previniendo el colapso de la vía aérea durante la espiración. } \\
\text { Recomendado en fibrosis quística sobre fisioterapia pulmonar convencional. Grado de recomendación, B }\end{array}$ \\
\hline $\begin{array}{l}\text { Mechanical Insufflation- } \\
\text { Exsufflation (MI-E). Cough } \\
\text { Assist Respironics }\end{array}$ & $\begin{array}{l}\text { Insuflación-exuflación mecánicamente asistida. Simula la tos mediante la aplicación de una presión positiva a } \\
\text { la vía aérea que cambia rápido a una presión negativa, permitiendo el drenaje de secreciones. Usado principal- } \\
\text { mente en enfermedades neurodegenerativas o secuelas neurológicas. Grado de recomendación, C }\end{array}$ \\
\hline $\begin{array}{l}\text { Intrapulmonary Percussive } \\
\text { Ventilation (IPV) Percussio- } \\
\text { naire }\end{array}$ & $\begin{array}{l}\text { Ventilación percusiva intrapulmonar. Ventilación oscilatoria de alta frecuencia para producir «percusión" a través } \\
\text { de liberación de descargas de gas a flujo alto directo a las vías aéreas. Buena alternativa para fibrosis Quística. } \\
\text { Grado de recomendación, I }\end{array}$ \\
\hline $\begin{array}{l}\text { High-frequency chest wall } \\
\text { compression devices. Vest }{ }^{T M} \\
\text { Airway Clearance System }\end{array}$ & $\begin{array}{l}\text { Compresión de alta frecuencia del tórax. Chaleco inflable con mangueras conectadas a un generador de pulsos } \\
\text { de aire. Se desinfla e infla liberando la pared del tórax de } 5 \text { a 20 veces por segundo creando un flujo de aire } \\
\text { dentro de los pulmones que despega las secreciones. Puede ser buena alternativa para fibrosis quística. Grado } \\
\text { de recomendación, I }\end{array}$ \\
\hline
\end{tabular}

de $\mathrm{SS} 0.9 \%$. Entre ellos se ha reportado el compromiso de las propiedades antimicrobianas de las secreciones en la vía aérea (nasales, tráquea y bronquios) ocasionado por las altas concentraciones de cloro y sodio. ${ }^{29-31}$ Con base en lo anterior, Christensen y colegas desarrollaron una solución baja en sodio y cloro para el manejo de secreciones en $\mathrm{UCI}$ neonatales. Los resultados más importantes mostraron que el empleo de dicha solución se asocia con una menor incidencia de neumonía asociada a ventilación mecánica al ser comparada con el uso de SS0.9\%. Sin embargo, los autores enfatizan que se requieren más estudios para controlar confusores y corroborar este hallazgo. ${ }^{32}$

Otro aspecto para considerar es la recuperación del volumen de SS0.9\% luego de administrarlo en un procedimiento de aspiración endotraqueal. En los escenarios clínicos es común tener la concepción que se logra recuperar el volumen instilado durante el procedimiento. No obstante, Hanley y su equipo demostraron con un estudio con $\mathrm{SS} 0.9 \%$ radiomarcada que únicamente se logra recuperar entre $11 \%$ y $19 \%$ del total de volumen administrado a la vía respiratoria a través de una cánula orotraqueal. ${ }^{28}$ El líquido no recuperado puede interferir con el intercambio de oxígeno alveolocapilar. Esta descripción ha permitido conocer los efectos del remanente no recuperado. El desarrollo de hipoxemia representa uno de los efectos no deseados con la administración de SSO.9\%. Ackerman y colaboradores analizaron el comportamiento de la saturación de oxígeno luego de administrar 5 $\mathrm{ml}$ de SS $0.9 \%$ previo a la succión endotraqueal. ${ }^{33}$ Los resultados fueron contundentes al evidenciar la presencia de hipoxemia en el minuto 2, 3, 4 y 5 posterior a la succión. Asimismo, se ha informado que el efecto puede persistir hasta 10 minutos luego de la succión endotraqueal..$^{34}$ Los resultados se han reproducido en pacientes pediátricos con mayor repercusión durante los primeros 2 minutos posterior a la succión, independientemente de administrar oxígeno al $100 \%$ previo al procedimiento. ${ }^{28}$

Los hallazgos mostrados forman parte de la estrategia actual para disminuir el uso de SS $0.9 \%$ previo a la succión endotraqueal. En paralelo, fomentar el incremento en el uso de sistemas de humedad que proporcionen un adecuado nivel de hidratación en la vía aérea; de forma subsecuente, mejorar el manejo de secreciones.

\section{MANEJO NO FARMACOLÓGICO}

\section{Succión traqueal}

La presencia de un dispositivo en la vía aérea (v.gr., tubo orotraqueal) compromete el aclaramiento mucociliar; asimismo, la adición de presión positiva durante el manejo con ventilación mecánica pueden favorecer que disminuya el tránsito del moco hacia la orofaringe. ${ }^{35}$ Luego entonces, el moco colectado en la vía aérea inferior requiere ser extraído mediante técnicas de succión para prevenir complicaciones (obstrucción de la vía aérea, aumento en el trabajo respiratorio, deterioro en el intercambio gaseoso e inestabilidad hemodinámica). ${ }^{5}$

La succión traqueal representa el estándar del manejo de pacientes que se encuentran bajo ventilación mecánica, empero, no es inocua; entre las complicaciones se encuentran: trauma mecánico, hipoxemia, broncoespasmo e inestabilidad hemodinámica. ${ }^{5,28,33} \mathrm{El}$ desarrollo de complicaciones es proporcional al número de eventos de succión. En la actualidad se carecen de lineamientos referentes a las técnicas y tiempos adecuados para lle- 
Neumol Cir Torax. 2019; 78 (3): 313-323

var a cabo el procedimiento. Las guías mejor adaptadas derivan de la Asociación Americana de Cuidados Respiratorios (AARC, por sus siglas en inglés), ${ }^{36}$ y sugieren que la succión traqueal debe ser realizada con la menor frecuencia posible o en casos debidamente indicados (acumulo evidente de secreciones). Con el objetivo de mejorar los tiempos de succión, recién se ha desarrollado un dispositivo acústico que permite identificar el acumulo de secreciones en la vía aérea (TBA Care ${ }^{\mathrm{T}}$, First Medica, Italy). Esta innovación potencialmente mejorará el manejo de secreciones mediante la succión traqueal. Lucchini y colegas compararon su eficacia contra las indicaciones estandarizadas (un procedimiento por turno a cargo del departamento de enfermería) y evidenciaron menor frecuencia de aspiraciones con el uso del sensor acústico; asimismo, se documentó que $48 \%$ de las aspiraciones programadas (por turno) no extrajeron secreciones. ${ }^{5}$ En conclusión, este dispositivo parece proporcionar una indicación más confiable en la succión traqueal. No obstante, se requieren de ensayos subsecuentes que ratifiquen su utilidad.

\section{Dispositivos mecánicos}

Se han investigado múltiples dispositivos de asistencia en el manejo de las secreciones bronquiales (tabla 2). Cabe destacar que la mayoría de los estudios se han realizado en pacientes con FQ, evidenciando el beneficio de los dispositivos en el manejo de las secreciones al ser comparado con la fisioterapia convencional. ${ }^{37} \mathrm{~A}$ continuación se detallan los más comunes.

\section{Presión positiva espiratoria}

PEP (Positive Expiratory Pressure por sus siglas en inglés). Acorde con la información actual, se ha establecido que aplicar una presión entre +5 y $+20 \mathrm{cmH}_{2} \mathrm{O}$ a través de una mascarilla facial mejora el drenaje de las secreciones. Entre los mecanismos propuestos se incluyen: a) aumento de la presión intratorácica distal a las secreciones mediante ventilación colateral o aumento en la capacidad funcional residual, y b) prevención del colapso en la vía aérea al generar un efecto de «férula» durante la espiración. ${ }^{38,39}$ Una revisión sistematizada que comparó PEP con fisioterapia pulmonar en pacientes con FQ mostró que no había diferencias significativas sobre el drenaje de las secreciones y el volumen espiratorio forzado del primer segundo $\left(\mathrm{VEF}_{1}\right)$ durante tres meses de seguimiento. Sin embargo, los pacientes prefirieron el PEP. ${ }^{40}$

Los resultados sobre la utilidad en otras enfermedades respiratorias v.gr., bronquitis crónica, no son contundentes para emitir una recomendación; por lo tanto, la sugerencia actual está dirigida a pacientes con FQ. ${ }^{41}$

\section{Tos mecánicamente asistida}

El mecanismo de la tos consta de 3 fases: 1) Fase inspiratoria: comienza con una inspiración profunda precedida por la apertura de la glotis, desplazamiento del diafragma y aumento en el diámetro de la caja torácica (aumento del volumen pulmonar); 2) Fase compresiva: caracterizada por el cierre de la glotis, seguido por relajación del diafragma y contracción violenta de los músculos espiratorios (aumento considerable de la presión intratorácica) que culmina con la apertura súbita de la glotis; 3) Fase explosiva: inicia con la apertura brusca de la glotis; la diferencia de presión que se desarrolla en la vía aérea genera un flujo acelerado culminando con la salida explosiva de aire. La fuerza de arrastre expulsa las partículas extrañas y el moco. ${ }^{42}$

Los pacientes que manifiestan debilidad de los músculos respiratorios tienen tos ineficiente..$^{37}$ La máquina de la tos o cough-assist (mechanical in-exufflation) reproduce la fisiología normal de la tos y facilita el drenaje de secreciones bronquiales. Adiciona presiones positivas (fase inspiratoria) que oscilan entre +30 y $+50 \mathrm{cmH}_{2} \mathrm{O}$ combinadas con presiones negativas (fase explosiva) que oscilan entre -20 y $-40 \mathrm{cmH}_{2} \mathrm{O}$. Asimismo, programa los tiempos inspiratorio (2-4 segundos) y de exhalación (4-5 segundos). ${ }^{43}$ Puede utilizarse con máscara oronasal, boquilla con clip nasal y a través de las cánulas de traqueostomía. ${ }^{44}$ En pacientes con enfermedad neuromuscular ha demostrado mejoría en el flujo pico de la tos alcanzando valores cuatro veces superior al valor basal, con flujos que oscilan entre 6 y $11 \mathrm{l} / \mathrm{seg}$ (valor normal: > $12 \mathrm{l} / \mathrm{seg}$.). ${ }^{37,43}$ En adición, el manejo de secreciones con esta estrategia se asocia con menor número de ingresos hospitalarios por paciente/año y menor tiempo de estancia en hospital. ${ }^{43,45}$ El enfoque actual está dirigido

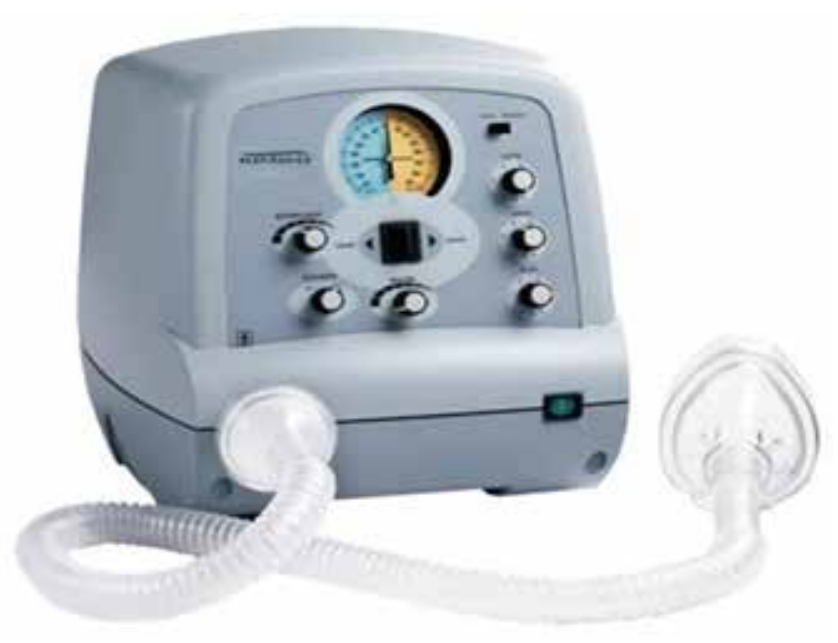

Figura 2: Máquina de tos. 


\section{Método de oscilación de alta frecuencia}

Consiste en un chaleco inflable que se conecta a un generador de impulsos de aire. El mecanismo de acción es inflar y desinflar, gentil y rápidamente para comprimir y liberar la pared del tórax; la frecuencia de eventos se sitúa entre 5 y 20 ciclos por segundo con el propósito de crear un flujo de aire en el interior de la vía aérea. Este mecanismo permite despegar el moco para ser movilizado hacia la vía aérea central de tal forma que puedan ser expulsadas por mecanismos como la tos o la succión. ${ }^{37}$

Hasta el momento, los estudios que han evaluado la asociación entre la oscilación del tórax y el drenaje de secreciones son poco concluyentes. Los efectos más notables

Figura 3:

Equipo de ventilación con percusión intrapulmonar.

a los pacientes con destete de la ventilación mecánica donde parece incidir en prevención de falla respiratoria postextubación y enfermedades neuromusculares ${ }^{46}$ (figura 2).

\section{Ventilación con percusión intrapulmonar}

$I P V$, por sus siglas en inglés, es una técnica que utiliza ventilación oscilatoria de alta frecuencia para producir «percusión» endotraqueal. ${ }^{37}$ El mecanismo de acción consiste en liberar corrientes de gas con flujos altos en la vía aérea a una frecuencia entre 100 y 300 impulsos por minuto controlados mediante un interruptor llamado Phasitron. La relación entre los impulsos y la exhalación genera la presión intrapulmonar en cuña, aspecto crucial en proporcionar la movilización óptima y el drenaje de las secreciones. ${ }^{47} \mathrm{En}$ paralelo, mejora la eficiencia y distribución de la ventilación, proporciona un medio alterno para administrar broncodilatadores y presión positiva intratorácica. Puede aplicarse mediante dispositivos orales, mascarillas, traqueostomía o tubo orotraqueal. ${ }^{36}$

La utilidad clínica se ha demostrado en pacientes con exacerbación de EPOC al ser comparado con fisioterapia pulmonar percusiva. Vargas y colaboradores informaron una mejoría de la frecuencia respiratoria, incremento de presión parcial de oxígeno y menor tiempo de estancia hospitalaria. ${ }^{48}$ Asimismo, se ha empleado en pacientes liberados del ventilador con traqueostomía demostrando mejoría en el índice de Kirby y en la fuerza muscular. ${ }^{49} \mathrm{El}$ empleo de la estrategia en pacientes con FQ disminuye la hiperinflación dinámica, mejora la mecánica ventilatoria y la proporción ventilación/perfusión ${ }^{50}$ (figura 3). se han informado en pacientes con FQ, dado que facilita la movilización de secreciones, incluso después de la primera hora de tratamiento al ser comparado con la fisioterapia convencional. ${ }^{50}$ Yuan y Dosman han empleado esta tecnología en pacientes pediátricos con problemas neurológicos (v.gr., parálisis cerebral y enfermedades neuromusculares); han informado un beneficio con menos fatiga y estancia hospitalaria con adecuada tolerancia. Otros escenarios clínicos están en proceso de ser analizados ${ }^{51,52}$ (figura 4).

\section{Aerosolterapia}

Principios generales

La aerosolterapia es una modalidad de tratamiento que se basa en la administración de sustancias en forma de aerosol por vía inhalatoria..$^{53}$ Un aerosol es una suspensión estable de partículas sólidas o líquidas en aire u otro gas, como el oxígeno. La ventaja principal de la aerosolterapia

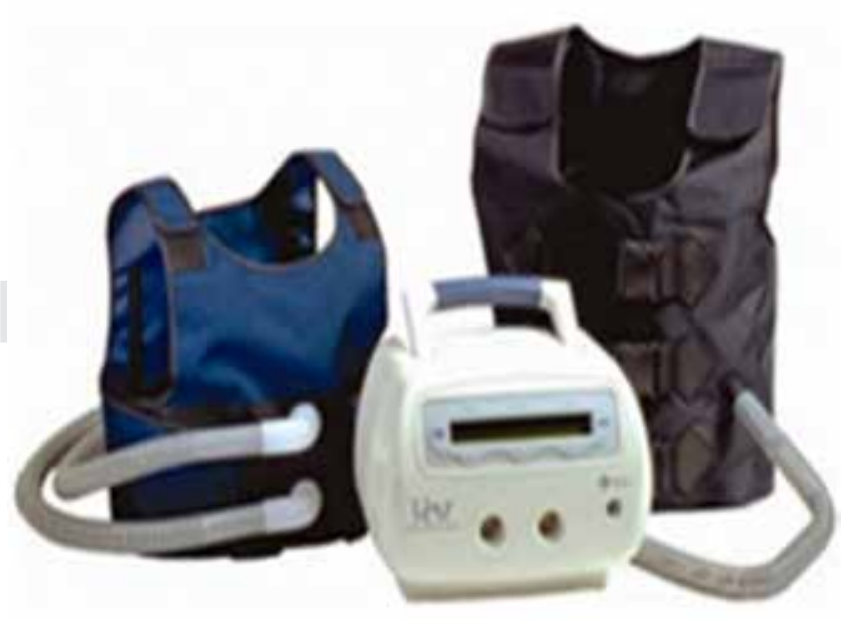

Figura 4: Dispositivos para técnica de oscilación de alta frecuencia del tórax (chaleco percutor). 
Neumol Cir Torax. 2019; 78 (3): 313-323

es que el aerosol se deposita de forma directa en el tracto respiratorio, con lo que pueden alcanzarse concentraciones mayores de la sustancia aerosolizada en el árbol bronquial y lecho pulmonar con menores efectos secundarios que si se utilizase la vía sistémica. En el año 2001, se publicaron los lineamientos más consistentes sobre el uso de nebulizadores recomendándolos en las siguientes situaciones: 1) pacientes que requieren dosis altas de broncodilatadores; 2) enfermos que precisan inhalar fármacos que sólo existen en esa presentación (DNasa o antibióticos en aerosol), y 3) pacientes incapaces de utilizar otros dispositivos de inhalación. ${ }^{54} \mathrm{Si}$ bien, los aerosoles ideales deberían ser monodispersos con partículas de tamaño y forma similares, la realidad es que son heterodispersos, es decir, formados por un conjunto de partículas de diversos diámetros. Los factores determinantes del tamaño de la partícula producida por un nebulizador incluyen tanto las características de la solución (densidad, viscosidad y tensión superficial de la solución nebulizada) como la velocidad de flujo del sistema de nebulización..$^{55} \mathrm{El}$ tamaño de las partículas generadas es inversamente proporcional al flujo del aerosol (tabla 3). Las leyes físicas intervienen en el depósito de las partículas, y esto se logra mediante tres mecanismos principales:

Impactación. Se presenta con cambios de flujo a gran velocidad, como ocurre en la bifurcación de las vías aéreas grandes y en las zonas con turbulencias. Ocurre en partículas grandes mayores a 5 micras de diámetro.

Sedimentación: Fenómeno físico a través del cual las partículas de un aerosol se depositan en las paredes de la vía aérea por acción de la gravedad. Constituye mecanismo principal de depósito en los bronquios distales y de pequeño diámetro. Se favorece con flujos bajos y un tamaño de partícula entre 2 y 5 micras de diámetro.

Difusión: Fenómeno que consiste en un movimiento aleatorio de las partículas inhaladas dentro de la vía aérea. Tiene menor importancia terapéutica pues se expulsa por depuración mucociliar o fagocitosis por parte de los macrófagos debido a su pequeño tamaño menor a 2 micras.

Tabla 3: Depósito de aerosoles según el tamaño de partícula.

\begin{tabular}{|c|l|}
\hline Tamaño & \multicolumn{1}{|c|}{ Depósito, eficacia y seguridad } \\
\hline$>5 \mu m$ & $\begin{array}{l}\text { Boca, tráquea y bronquios principales. Sin } \\
\text { efecto terapéutico. Absorción vía digestiva y } \\
\text { eliminación mucociliar }\end{array}$ \\
\hline $1-5 \mu m$ & $\begin{array}{l}\text { Vías aéreas superiores. Efecto terapéutico. } \\
\text { Absorción desde el pulmón }\end{array}$ \\
\hline$<1 \mu m$ & $\begin{array}{l}\text { Alvéolos. Sin efecto terapéutico. Eliminación } \\
\text { exhalada }\end{array}$ \\
\hline
\end{tabular}

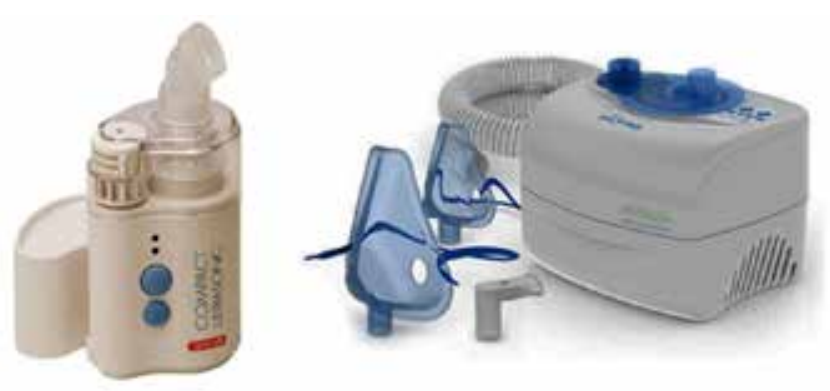

Figura 5: Nebulizadores ultrasónicos.

\section{Sistemas de nebulización}

Estos dispositivos se componen de una cámara de nebulización donde se introduce el líquido a nebulizar que será transformado en un aerosol y de un generador de energía. Existen tres tipos de sistemas de nebulización: nebulizadores ultrasónicos, jet (también denominados como neumáticos o de chorro de aire) y de malla o electrónicos. Para inhalar el aerosol de la cámara de nebulización se precisa una interfase (pieza bucal, mascarilla nasobucal, conexión en $\mathrm{T}$ a la tráquea, mascarilla traqueal u horquilla nasal). Cabe señalar que la mascarilla nasobucal sólo se recomienda en los menores de seis años o cuando el paciente no puede o no sabe utilizar la pieza bucal. La elección de la interfase se individualiza a las características del paciente. ${ }^{53}$

\section{Nebulizadores ultrasónicos}

Los nebulizadores ultrasónicos utilizan como fuente de energía la vibración de alta frecuencia a través de un cristal piezoeléctrico. Las vibraciones del cristal producen oscilaciones en el líquido, dando lugar a su nebulización. El tamaño de las partículas es directamente proporcional a las vibraciones. Producen flujos entre 2 y $20 \mathrm{l} / \mathrm{min}$ y tienen capacidad para nebulizar grandes volúmenes; sin embargo, no son apropiados para la nebulización de antibióticos ni fármacos como los corticoesteroides y la DNasa dado que, las ondas de alta frecuencia se disipan en forma de calor lo que puede afectar a la estabilidad de la suspensión. Pueden utilizarse para nebulizar soluciones con broncodilatadores o suero salino ${ }^{53}$ (figura 5).

\section{Nebulizadores jet}

Los nebulizadores jet están conformados por una cámara de nebulización, donde se introduce el líquido a nebulizar y una fuente de energía. ${ }^{53}$ En estos dispositivos, el aerosol se genera con el impacto del flujo de gas (aire u oxígeno) en la cámara del nebulizador. Al aumentar la velocidad del fluido disminuye su presión (efecto Venturi) generando un 
gradiente de presión negativa por encima del orificio superior del tubo capilar lo que hace que el líquido ascienda (efecto Bernoulli) y choque contra un sistema de percusión fragmentándose en múltiples y pequeñas gotas. Una pantalla permite por impacto que las gotas mayores vuelvan al reservorio, en tanto, las más pequeñas pueden ser inhaladas. Como fuente de energía se utiliza un compresor mecánico de aire o gas comprimido (oxígeno o aire). Hay varios tipos de nebulizadores jet, clasificados según su funcionamiento durante la inhalación. Los más utilizados son los nebulizadores convencionales con débito constante, los nebulizadores con efecto Venturi activo durante la inspiración y los dosimétricos (también denominados nebulizadores jet con sistema de dosificación en la inspiración o nebulizadores jet con liberación adaptada de aerosol). Como norma general, al utilizar un sistema jet se recomienda emplear un compresor con alto flujo dinámico y un nebulizador que genere más del $70 \%$ de las partículas respirables en el menor tiempo posible (10-15 $\mathrm{min})^{55}$ (figura 6).

\section{Nebulizadores de malla}

En este sistema de nebulización, el aerosol se genera al pasar la solución a nebulizar por los agujeros de una malla. No necesitan compresor, son menos pesados y ruidosos que los jet. Además de funcionar con electricidad, pueden funcionar con pilas y con la batería del coche. Hay dos tipos de nebulizadores de malla: estática y vibratoria. En los de malla estática, el aerosol se genera aplicando una presión en el líquido para que pase a través de los orificios de la malla. Por otro lado, en el tipo malla vibratoria, el líquido pasa por los agujeros gracias a la vibración de la malla. La eficacia de estos sistemas es superior al nebulizador tipo jet, al favorecer un mayor depósito pulmonar, del mismo modo, son menos voluminosos, más silenciosos y más rápidos, lo que se traduce en un mejor cumplimiento por parte del

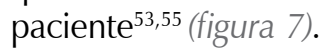

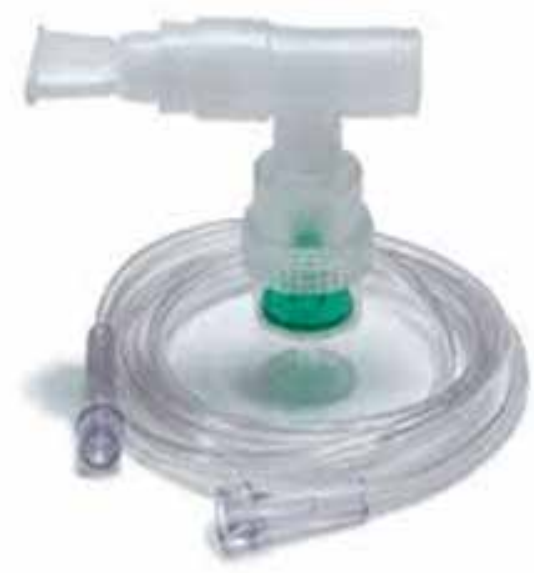

Figura 6:

Nebulizador de Jet.
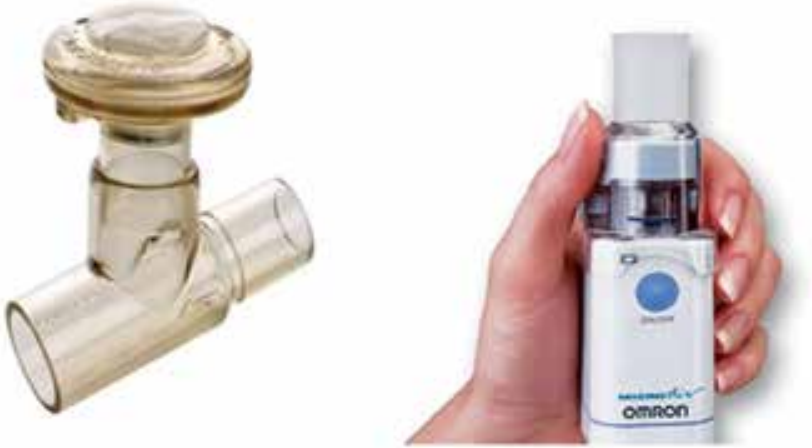

Figura 7: Nebulizadores de malla vibrante.

\section{CONCLUSIONES}

Las estrategias de fisioterapia respiratoria han estado sufriendo un cambio continuo en los últimos años. El efecto a largo plazo de las técnicas no farmacológicas del manejo de secreciones sobre la calidad de vida, tasas de exacerbaciones, hospitalizaciones y mortalidad, son favorables; no obstante, es necesario realizar estudios que involucren subpoblaciones de pacientes para generalizar los resultados. A pesar de los límites, algunas técnicas están bien documentadas en pacientes con FQ.

Las terapias actuales para el manejo de secreciones están enfocadas en reducir la inflamación e inducir la broncodilatación y son pocos los datos relacionados al control de la hipersecreción de moco en las vías respiratorias. Este límite representa una necesidad no resuelta para desarrollar compuestos farmacológicamente activos encaminados a exceso de síntesis y secreción de mucina.

Por último, el conocimiento de los principios generales de la aerosolterapia, los fármacos diseñados para ello, la dinámica de las partículas, las singularidades de las soluciones para nebulizar y las modalidades de nebulización harán que este arsenal terapéutico permee más en el colectivo del personal de la salud con el fin de optimizar recursos, aplicar de manera correcta el tratamiento para cada paciente en particular y brindar la mayor probabilidad de éxito en el manejo médico.

\section{REFERENCIAS}

1. Jelic S, Cunningham JA, Factor P. Clinical review: airway hygiene in the intensive care unit. Crit Care 2008;12(2):209. https://doi. org/10.1186/cc6830

2. Voynow JA, Rubin BK. Mucins, mucus, and sputum. Chest 2009;135(2):505-512. https://doi.org/10.1378/chest.08-0412

3. Balsamo R, Lanata L, Egan CG. Mucoactive drugs. Eur Respir Rev 2010;19(116):127-133. https://doi.org/10.1183/09059180.00003510

4. Hess DR. Airway clearance: physiology, pharmacology, techniques, and practice. Respir Care 2007;52(10):1392-1396. 
5 Lucchini A, Zanella A, Bellani G, et al. Tracheal secretion management in the mechanically ventilated patient: comparison of standard assessment and an acoustic secretion detector. Respir Care 2011;56(5):596-603. https://doi.org/10.4187/ respcare.00909

6. Rogers DF, Barnes PJ. Treatment of airway mucus hypersecretion. Ann Med 2006;38(2):116-125. https://doi.org/10.1080/07853890600585795

7. Rubin BK. Mucolytics, expectorants, and mucokinetic medications. Respir Care 2007;52(7):859-865.

8. Yuta A, BaraniukJN. Therapeutic approaches to mucus hypersecretion. Curr Allergy Asthma Rep 2005;5(3):243-251.

9. Rubin BK. An in vitro comparison of the mucoactive properties of guaifenesin, iodinated glycerol, surfactant, and albuterol. Chest 1999;116(1):195-200. https://doi.org/10.1378/chest.116.1.195

10. Rubin BK, Ramirez O, Ohar JA. lodinated glycerol has no effect on pulmonary function, symptom score, or sputum properties in patients with stable chronic bronchitis. Chest 1996;109(2):348-352. https://doi. org/10.1378/chest.109.2.348

11. Shim C, King M, Williams MH Jr. Lack of effect of hydration on sputum production in chronic bronchitis. Chest 1987;92(4):679-682. https:// doi.org/10.1378/chest.92.4.679

12. Dekhuijzen PN. Antioxidant properties of $\mathrm{N}$-acetylcysteine: their relevance in relation to chronic obstructive pulmonary disease. Eur Respir J 2004;23(4):629-636.

13. Felton VM, Borok Z, Willis BC. N-acetylcysteine inhibits alveolar epithelial-mesenchymal transition. Am J Physiol Lung Cell Mol Physiol 2009;297(5):L805-L812. https://doi.org/10.1152/ ajplung.00009.2009

14. Decramer M, Rutten-van Mölken M, Dekhuijzen PN, et al. Effects of $\mathrm{N}$-acetylcysteine on outcomes in chronic obstructive pulmonary disease (Bronchitis Randomized on NAC Cost-Utility Study, BRONCUS): a randomised placebo-controlled trial. Lancet 2005;365(9470):1552-1560. https://doi.org/10.1016/S01406736(05)66456-2

15. Hirai DM, Jones JH, Zelt JT, et al. Oral N-acetylcysteine and exercise tolerance in mild chronic obstructive pulmonary disease. JAppl Physiol (1985) 2017;122(5):1351-1361. https://doi.org/10.1152/ japplphysiol.00990.2016

16. Ehre C, Rushton ZL, Wang B, et al. An improved inhaled mucolytic to treat airway muco-obstructive diseases. Am J Respir Crit Care Med 2019;199(2):171-180. https://doi.org/10.1164/rccm.201802$02450 \mathrm{C}$

17. McPhail GL, Acton JD, Fenchel MC, Amin RS, Seid M. Improvements in lung function outcomes in children with cystic fibrosis are associated with better nutrition, fewer chronic pseudomonas aeruginosa infections, and dornase alfa use. J Pediatr 2008;153(6):752-757. https://doi.org/10.1016/j.jpeds.2008.07.011

18. Arcuri JF, Abarshi E, Preston NJ, Brine J, Pires Di Lorenzo VA. Benefits of interventions for respiratory secretion management in adult palliative care patients-a systematic review. BMC Palliat Care 2016;15:74. https://doi.org/10.1186/s12904-016-0147-y

19. Ha EV, Rogers DF. Novel therapies to inhibit mucus synthesis and secretion in airway hypersecretory diseases. Pharmacology 2016;97(1-2):84-100. https://doi.org/10.1159/000442794

20. Fuloria M, Rubin BK. Evaluating the efficacy of mucoactive aerosol therapy. Respir Care 2000;45(7):868-873.

21. Martinez FJ, Curtis JL, Albert R. Role of macrolide therapy in chronic obstructive pulmonary disease. Int J Chron Obstruct Pulmon Dis 2008;3(3):331-350.
22. Gotfried MH. Macrolides for the treatment of chronic sinusitis, asthma, and COPD. Chest 2004;125(2 Suppl):52S-60S. https://doi. org/10.1378/chest.125.2_suppl.52s

23. Albert RK, Connett J, Bailey WC, et al:; COPD Clinical Research Network. Azithromycin for prevention of exacerbations of COPD. N Engl J Med 2011;365(8):689-698. https://doi.org/10.1056/ NEJMoa1104623

24. Malerba M, Ragnoli B. Ambroxol in the 21st century: pharmacological and clinical update. Expert Opin Drug Metab Toxicol 2008;4(8):11191129. https://doi.org/10.1517/17425255.4.8.1119

25. Cortes-Telles A, García-Torrentera R. Aerosoles: Fármacos y los dispositivos para suadministración. Neumol Cir Torax 2013;72(2):164-174.

26. Witek TJ Jr. The fate of inhaled drugs: the pharmacokinetics and pharmacodynamics of drugs administered by aerosol. Respir Care 2000;45(7):826-830.

27. Colice GL. Comparing inhaled corticosteroids. Respir Care 2000;45(7):846-853.

28. Ridling DA, Martin LD, Bratton SL. Endotracheal suctioning with or without instillation of isotonic sodium chloride solution in critically ill children. Am J Crit Care 2003;12(3):212-219.

29. Ganz T. Antimicrobial polypeptides in host defense of the respiratory tract. J Clin Invest 2002;109(6):693-697. https://doi.org/10.1172/JCl15218

30. Herr C, Shaykhiev R, Bals R. The role of cathelicidin and defensins in pulmonary inflammatory diseases. Expert Opin Biol Ther 2007;7(9):1449-1461. https://doi.org/10.1517/14712598.7.9.1449

31. Travis SM, Conway BA, Zabner J, et al. Activity of abundant antimicrobials of the human airway. Am J Respir Cell Mol Biol 1999;20(5):872-879.

32. Christensen RD, Henry E, Baer VL, et al. A low-sodium solution for airway care: results of a multicenter trial. Respir Care 2010;55(12):1680-1685.

33. Ackerman MH. The effect of saline lavage prior to suctioning. Am J Crit Care 1993;2(4):326-330.

34. Ackerman MH, Mick DJ. Instillation of normal saline before suctioning in patients with pulmonary infections: a prospective randomized controlled trial. Am J Crit Care 1998;7(4):261-266.

35. Li Bassi G, Zanella A, Cressoni M, Stylianou M, Kolobow T. Following tracheal intubation, mucus flow is reversed in the semirecumbent position: possible role in the pathogenesis of ventilator-associated pneumonia. Crit Care Med 2008;36(2):518-525. https://doi. org/10.1097/01.CCM.0000299741.32078.E9.

36. American Association for Respiratory C. AARC Clinical Practice Guidelines. Endotracheal suctioning of mechanically ventilated patients with artificial airways 2010. Respir Care 2010;55(6):758-764.

37. McCool FD, Rosen MJ. Nonpharmacologic airway clearance therapies: ACCP evidence-based clinical practice guidelines. Chest 2006;129(1 Suppl):250S-259S. https://doi.org/10.1378/chest.129.1_ suppl.250S.

38. Groth S, Stafanger G, Dirksen H, Andersen JB, Falk M, Kelstrup M. Positive expiratory pressure (PEP-mask) physiotherapy improves ventilation and reduces volume of trapped gas in cystic fibrosis. Bull Eur Physiopathol Respir 1985;21(4):339-343.

39. Oberwaldner B, Evans JC, Zach MS. Forced expirations against a variable resistance: a new chest physiotherapy method in cystic fibrosis. Pediatr Pulmonol 1986;2(6):358-367.

40. Elkins MR, Jones A, van der Schans C. Positive expiratory pressure physiotherapy for airway clearance in people with cystic fibrosis. Cochrane Database Syst Rev 2006;(2):CD003147. https://doi. org/10.1002/14651858.CD003147.pub3. 
41. Christensen HR, Simonsen $K$, Lange $P$, et al. PEEP-masks in patients with severe obstructive pulmonary disease: a negative report. Eur Respir J 1990;3(3):267-272.

42. McCool FD. Global physiology and pathophysiology of cough: ACCP evidence-based clinical practice guidelines. Chest 2006;129(1 Suppl):48S-53S. https://doi.org/10.1378/chest.129.1_suppl.48S.

43. Homnick DN. Mechanical insufflation-exsufflation for airway mucus clearance. Respir Care 2007;52(10):1296-1305.

44. Bosch A, Winterholler M. [Technical aspects of mechanical insufflator-exsufflators. Construction and function of the Emerson CoughAssist]. Pneumologie 2008;62 Suppl 1:S49-S54. https://doi. org/10.1055/s-2007-1016441.

45. Chatwin M, Simonds AK. The addition of mechanical insufflation/ exsufflation shortens airway-clearance sessions in neuromuscular patients with chest infection. Respir Care 2009;54(11):1473-1479.

46. Gonçalves MR, Honrado T, Winck JC, Paiva JA. Effects of mechanical insufflation-exsufflation in preventing respiratory failure after extubation: a randomized controlled trial. Crit Care 2012;16(2):R48. https://doi.org/10.1186/cc11249.

47. Chatburn RL. High-frequency assisted airway clearance. Respir Care 2007;52(9):1224-1235.

48. Vargas F, Bui HN, Boyer A, et al. Intrapulmonary percussive ventilation in acute exacerbations of COPD patients with mild respiratory acidosis: a randomized controlled trial [ISRCTN17802078]. Crit Care 2005;9(4):R382-R389. https://doi.org/10.1186/cc3724.

49. Clini EM, Antoni FD, Vitacca M, et al. Intrapulmonary percussive ventilation in tracheostomized patients: a randomized controlled trial. Intensive Care Med 2006;32(12):1994-2001. https://doi.org/10.1007/ s00134-006-0427-8.
50. Arens R, Gozal D, Omlin KJ, et al. Comparison of high frequency chest compression and conventional chest physiotherapy in hospitalized patients with cystic fibrosis. Am J Respir Crit Care Med 1994;150(4):1154-1157. https://doi.org/10.1164/ajrccm.150.4.7921452.

51. Dosman CF, Zuberbuhler PC, Tabak JI, Jones RL. Effects of positive end-expiratory pressure on oscillated volume during high frequency chest compression in children with cystic fibrosis. Can Respir J 2003;10(2):94-98. https://doi.org/10.1155/2003/792917.

52. Yuan N, Kane P, Shelton K, Matel J, Becker BC, Moss RB. Safety, tolerability, and efficacy of high-frequency chest wall oscillation in pediatric patients with cerebral palsy and neuromuscular diseases: an exploratory randomized controlled trial. J Child Neurol 2010;25(7):815821. https://doi.org/10.1177/0883073809350223.

53. Máiz Carro L, Wagner Struwing C. [Benefits of nebulized therapy: basic concepts]. Arch Bronconeumol 2011;47 Suppl 6:2-7. https:/l doi.org/10.1016/S0300-2896(11)70028-X.

54. Boe J, Dennis JH, O'Driscoll BR, et al.; European Respiratory Society Task Force on the use of nebulizers. European Respiratory Society Guidelines on the use of nebulizers. Eur Respir J 2001;18(1):228-242.

55. Laube BL, Janssens HM, de Jongh FH, et al:;European Respiratory Society; International Society for Aerosols in Medicine. What the pulmonary specialist should know about the new inhalation therapies. Eur Respir J 2011;37(6):1308-1331. https://doi. org/10.1183/09031936.00166410.

Conflicto de intereses: Los autores declaran no tener conflicto de intereses. 